\title{
Contrast optimization of two-photon processes after a microstructured hollow-core fiber demonstrated for dye molecules
}

\author{
Alexander Patas ${ }^{1}$, Georg Achazi ${ }^{1}$, Nils Hermes ${ }^{1}$, Monika Pawłowska $^{1}$, Albrecht Lindinger ${ }^{1}$ \\ Fachbereich Physik, Freie Universität Berlin, Arnimallee 14, 14195 Berlin, Germany, e-mail: patas@physik.fu-berlin.de
}

Received: date / Revised version: date

\begin{abstract}
We demonstrate selective excitation of dyes with overlapping absorption spectra in solution with pulses transmitted through a hollow-core fiber. Thereto we show how dispersive effects occurring in the fiber can be compensated and what the limiting pulse energies are. Furthermore, an overview over various phase parametrizations is given and we examine which are best used when optimizing a two-photon fluorescence contrast of two dyes in a sample. This could be relevant for future endoscopic applications as well as state of the art two-photon microscopy.
\end{abstract}

\section{Introduction}

Applying the ideas of coherent control to modern twophoton microscopy can lead to new insight on the chemical or structural composition of biological samples. Since two-photon absorption is a nonlinear process, it can reach a higher resolution than standard one-photon laser ex- citation microscopy [1]. Due to the infrared light used for excitation the penetration depth is greatly improved over one-photon fluorescence microscopy [2]. (* Marker substances are used to gain additional information about biological samples. For example Fura-2 AM is used neuroscience to detect Ca-Ion concentrations by two-photon laser scanning microscopy $[15,16]$ *) By tayloring the pulse's spectral phase and thereby taking advantage of intrapulse interference one can achieve excitations of narrow band transitions which are as efficient as the transformlimited pulse [3]. This effect also allows to reduce photodamage in a sample or to selectively excite different fluorophores with close lying excitation bands [4].

The aim to extract additional information out of twophoton excitation microscopy recently gave reason to different publications studying phase functions which efficiently excite different dye molecules selectively [5-8]. The question arises which contrast can be achieved and 
how much the fluorescence signal is being reduced in comparison to the transform-limited pulse.

When adequate pulse shapes are identified, the pulses have to be transported to the sample. (*This can be done via a single mode optical fiber while dispersion and nonlinear effects limit the delivered pulse intensities. Even a micro-optic scanning head was added to an endoscope showing the applicability of two-photon fluorescence endoscopy $[18,19]$. In contrast to standard single-mode fibers *) Hollow-core photonic crystal fibers favour the transmission of high pulse intensities required in two-photon excitation experiments due to their air filled core.

Compensating for effects in the linear and nonlinear regime as well as changes in birefringence by bending or twisting could in future enable an endoscopic application of (X such pulses $\mathrm{X})$ (* phase shaped pulses after optical fibers $*)[9,10]$.

(* Various fluorophores were examined [17] wich could be employed as markers in living cells. Unfortunately most two-photon laser scanning microscopes do not have the spectral resolution to distinguish between the dyes emission spectra.*) (*Additionally*) Biologically relevant fluorophores often do not have narrow absorption bands and thereby mostly overlap in the spectral region of the exciting laser pulse. In this paper, we want to identify which spectral phases are best used for effective selective excitation of dyes with overlapping absorption bands after transmission through a hollow-core

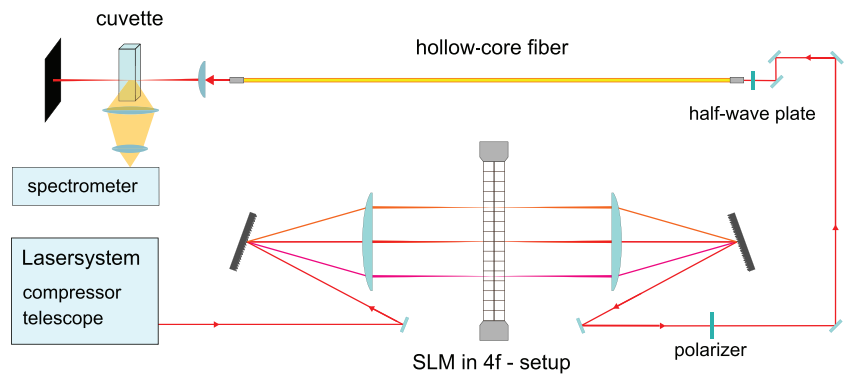

Fig. 1 Experimental setup used throughout this paper.

photonic crystal fiber. This is done by identifiying phaseparametrizations which are then used for compensation of the fiber's dispersive properties and optimization of the fluorescence contrast using an evolutionary optimization algorithm. (*Two allow for these optimizations in the full range of the examined phase functions we chose Rhodamine B and Coumarin 1 as an example system due to their high fluorescence efficiency. Every other dye could in principle be used in the same way while the efficiency of the phase functions would depend on the difference of the two-photon absorption spectra. We will show that the contrast enhancement due to phase shaping can be easily tested in prior to the experiment by simulating the contrast from the measured relative twophoton absorption of the applied dyes.*)

\section{Setup}

The experimental setup (Fig. 1) consists of a femtosecond oscillator (Mira) and a regenerative amplifier (RegA) delivering pulses at the rate of $286 \mathrm{kHz}$, with $805 \mathrm{~nm}$ central wavelength and at a spectral width of $26 \mathrm{~nm}$. After a grating compressor, pulse durations down to $60 \mathrm{fs}$ 
can be reached. These pulses are shaped by a Spatial Light Modulator (CRI SLM640) in the Fourier plane of a 4f-setup to achieve control over both spectral amplitude and phase. The SLM has 640 pixels with pixel number 321 being tuned to the central wavelength of $805 \mathrm{~nm}$ while its spectral resolution was determined to be $0.2715 \frac{\mathrm{nm}}{\mathrm{px}}$.

The pulses are then transmitted through a hollowcore photonic crystal fiber (HC-800-1 from NKT Photonics). In order to maximize the light coupled into the fiber we use a telescope after the pulse shaper to optimize the beam diameter at the input end. Even though hollowcore fibers are designed to allow for much higher pulse intensities than conventional optical fibers we have to reduce the laser power to $60 \mathrm{~mW}$ using an additional halfwave plate and polarizer in front of the fiber. Thereby we avoid damaging the front window of the hollow-core fiber which is essential as it prevents dust from entering the $9.5 \mu \mathrm{m}$ air filled hollow-core. This limits the maximal pulse energy in this experiment to $\sim 35 \mathrm{~nJ}$ (corresponding to $10 \mathrm{~mW}$ power after the hollow-core fiber). The fiber shows little dispersion and neglible nonlinear effects in the regime considered in this paper. The remaining dispersion is mainly a result of the waveguide properties of the fiber.

The beam diameter is then widened by a telescope and focused in a cuvette containing Rhodamine B and Coumarin 1 (also known as Coumarin 47 or Coumarin 460 ) in ethanol at concentrations of $25 \times 10^{-3}$ molar and
$7.5 \times 10^{-3}$ molar, respectively. When the pulses are transmitted by the fiber they are additionally distorted by chromatic dispersion. Therefore we had to apply a phase offset found by an evolutionary closed loop optimization of the pulse duration as described in [11]. This was done by maximizing the signal of a two-photon diode (G1116, Hamamatsu) with an absorption around $400 \mathrm{~nm}$ which favours short pulses due to the nature of broad twophoton transitions. By this method we found an optimal phase which gave us a transform-limited pulse at the cuvette's position (where the two-photon diode was located). The spectrum after the fiber was measured to ensure that there are no nonlinear effects present. Should higher pulse intensities be required, one can use the method of backpropagation [9] to calculate the compensated input pulse required for every desired spectral phase after the fiber. The intensities in our experiment were limited by the glass window sealing the front end of the hollow-core fiber which would get burned at high intensities due to the small effective area in which the laser-modes are confined. A different type of connector could in principle allow higher pulse intensities and would make the research on nonlinear effects in hollowcore fiber more accessible. Because the experiments in this paper take place in the linear regime neglecting the fiber's nonlinear effects, the phase found by the optimization can then be used as an offset which will be added to all desired phase functions discussed later in this work $[11,12]$. Finally, two lenses placed on an axis perpendic- 
ular to the exciting laser beam capture as much fluorescent light as possible to be analyzed by a spectrometer in the visible range.

The emission spectra of the two examined dyes are well separated ( ${ }^{*}$ while their absorption spectra overlap*). This allows us to compare the excitation efficiency by integrating the spectral intensity from $410 \mathrm{~nm}$ to $550 \mathrm{~nm}$ for Coumarin 1 and from $560 \mathrm{~nm}$ to $700 \mathrm{~nm}$ for Rhodamine B. The spectrometer data is collected and used as feedback for a closed loop optimization testing various parametric phase functions on their efficiency in exciting one of the two dyes selectively. The applied genetic optimization algorithm can be used to optimize any given fitness function. In this case we maximized or minimized the fluorescence contrast to reach maximal Rhodamine B and minimal Coumarin 1 fluorescence or maximal Coumarin 1 and minimal Rhodamine B fluorescence. Another approach to find optimal phase functions for selective excitation is to scan distinct parameters of the examined phase functions and measure the fluorescence contrast for each pulse shape. As the scan does not need to converge over many generations unlike a genetic optimization algorithm, it is considerably fast and provides good insight about the potential of special parametric phase functions.

\section{Simulations}

Two-photon processes are driven by the effective field resulting from the interference of different components within the pulse spectrum. The electric field in the spectral domain $|E(\omega)| e^{i \Phi(\omega)}$ consists of an amplitude and a phase factor which leads to the expression $E^{(2)}(2 \omega)=\int_{-\infty}^{\infty}|E(\omega-\Omega)||E(\omega+\Omega)| e^{i(\Phi(\omega-\Omega)+\Phi(\omega+\Omega))} d \Omega$

for the effective field driving the two-photon transition. If the spectral phase function $\Phi$ is antisymmetric around a chosen central wavelength $\omega_{c}$,

$$
\Phi\left(\omega_{c}-\omega\right)=-\Phi\left(\omega_{c}+\omega\right)
$$

the exponent in equation (1) vanishes and $E^{(2)}\left(2 \omega_{c}\right)$ achieves the same value as for the transform-limited pulse. For narrow two-photon transitions the excitation is proportional to $E^{(2)}(\omega)$. Therefore one can excite narrow two-photon transitions as efficient as the transform limited pulse with phase functions that are antisymmetric around a specific exitation wavelength (e.g.: step phase, sinus phase, third-order phase) [3].

For broad two-photon absorption as seen for Rhodamine B and Coumarin 1 the fluorescence intensity $I_{R / C}$ is proportional to

$$
I_{R / C} \sim \int_{-\infty}^{\infty}\left|E^{(2)}(\omega)\right|^{2} \cdot \operatorname{TPA}_{R / C}(\omega) d \omega
$$

where $\operatorname{TPA}_{R / C}(\omega)$ is the two-photon absorbance of Rhodamine B and Coumarin 1, respectively.

In order to simulate the fluorescence signal of the two dyes with broad absorption spectra we had to measure their relative two-photon absorption in a preliminary experiment. By amplitude shaping gaussian spectra with 

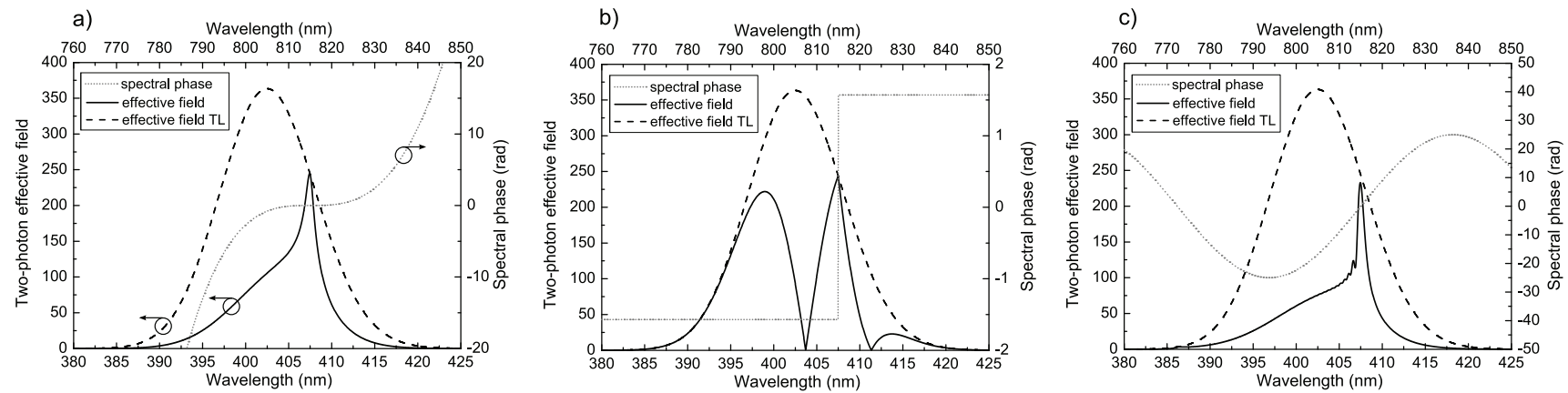

Fig. 2 Simulated two-photon field for three different phase functions. All functions are antisymmetric around $815 \mathrm{~nm}$ and show how interference can be used to selectively excite dyes absorbing at higher or lower wavelengths. The third-order phase (a) and sine phase (c) both result in a spike at $815 \mathrm{~nm}$ which will give large selectivity but low fluorescence signal. The $\pi$-step phase (b) features a peak at $815 \mathrm{~nm}$ as well but diminishes the two-photon field around the step position while leaving the spectrum elsewhere undisturbed. This should lead to larger fluorescence but overall less contrast.

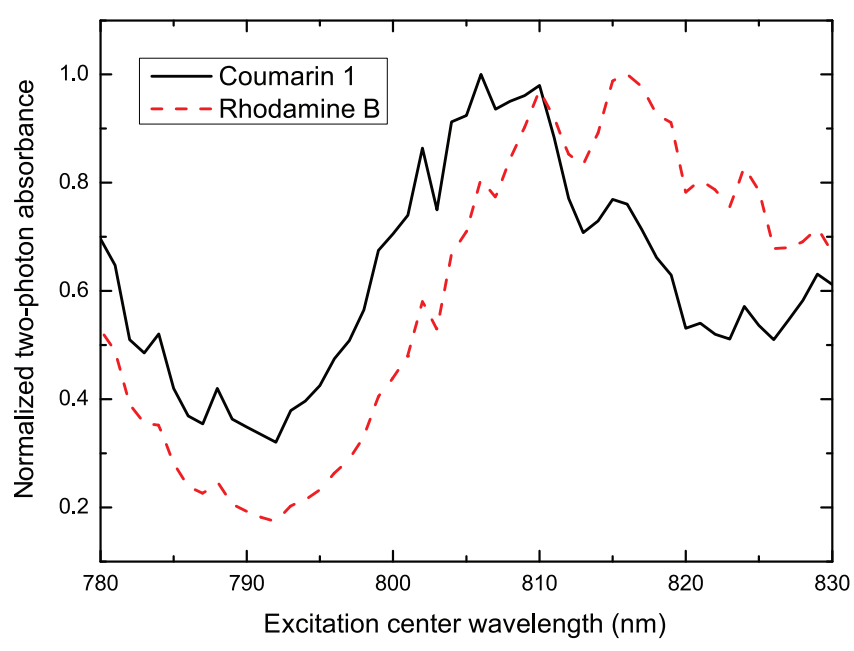

Fig. 3 Two-photon absorbance of Rhodamine B and Coumarin 1 measured in the spectral range of the exciting laser pulse. Amplitude-shaping was used to create transformlimited shifted gaussian pulses with a spectral width of 2.5 for each adressable central wavelength (x-axis). To approximate the two-photon absorbance the fluorescence spectra were integrated and the obtained values divided by the signal of a two-photon diode for normalization. shifted central wavelength were created and used to excite the solution in the full available spectral range to acquire the data shown in Fig. 3. Only the spectral range of our laser can be adressed by this method. This resulted in a growing uncertainty closer to the edge of the spectrum.

Our setup does not allow a measurement of the absolute two-photon absorption of our dyes. Favourably only the relative absorption is required when calculating the contrast. The contrast, often referred to in two-photon microscopy and biological imaging, is defined as

$$
C=\frac{I_{R}-I_{C}}{I_{R}+I_{C}} .
$$

In this case $I_{R}$ and $I_{C}$ are the fluorescence signals of Rhodamin B and Coumarin 1, respectively. The contrast can feature values from -1 to 1 when only Rhodamine $B$ or Coumarin 1 is excited while 0 stands for equally large fluorescence of both dyes. To account for the difference 
in fluorescence (*)(resulting from the difference in concentration $)^{*}$ ) when illuminated by a short pulse the calculated signal was normalized so that in the simulation the transform-limited pulse would result in a contrast of 0.18 just like in the experiment. Fluorescence intensities throughout this paper are defined as

$$
I_{f l}=\frac{1}{2}\left(\frac{I_{R}}{I_{R}^{T L}}+\frac{I_{C}}{I_{C}^{T L}}\right)
$$

where $I_{R}^{T L}$ and $I_{C}^{T L}$ are the integrated fluorescence values measured for the short pulse.

\section{Parameter scans}

From Fig. 3 we see that Coumarin 1 is better excited for wavelengths below the central wavelength of our laser, while the maximum absorption of Rhodamine B lies at higher wavelengths. An optimal phase for exiciting Rhodamine B and Coumarin 1 selectively would interfere constructively for one side and destructively for the other side of the spectrum.

All encodings shown in Table 1 were chosen to have a point of symmetry $\left(\lambda_{C}, \omega_{C}\right)$ and thereby allow constructive interference at this wavlength. In Fig. 2 this symmetry was tuned to $815 \mathrm{~nm}$ to demonstrate the effective field seen by two-photon transitions (Eq. 1) for such shifted spectral phase functions. The sine and thirdorder phase functions lead to mostly destructive interference for other wavelengths than $815 \mathrm{~nm}$, while the $\pi$-step phase exhibits constructive interference also away from
Table 1 Phase functions used in the scans. The phase was modulated by changing $\lambda_{c}$ or $\omega_{c}$ respectively and thus the point of symmetry. Parameter values used in the scans are shown in the third column. The function $\Theta(x)$ used here is also known as the Heaviside step-function.

\begin{tabular}{lll}
\hline phase function & parametrization & parameters \\
\hline sine & $A \sin \left(k\left(\lambda-\lambda_{c}\right)\right)$ & $A=25 \mathrm{rad}$, \\
& & $k=0.0736 \mathrm{~nm}^{-1}$ \\
third-order & $\frac{b_{3}}{6}\left(\omega-\omega_{c}\right)^{3}$ & $b_{3}=2 \times 10^{5} \mathrm{fs}^{3}$ \\
$\pi$-step & $\pi \Theta\left(\omega-\omega_{c}\right)$ & \\
\hline
\end{tabular}

the center of symmetry. As the $\pi$-step shifts to one side, the phase is flat for most of the spectrum and therefore the second-order field of the shaped pulse resembles the signal of the transform-limited pulse.

While shifting the spectral phase function pixelwise from $775 \mathrm{~nm}$ to $835 \mathrm{~nm}$ the spectral peaks of Rhodamine B and Coumarin 1 were recorded, integrated, and their contrast was computed. Fig. 4 shows the contrast plotted over the wavelength around which the shifted spectral phase is antisymmetric. Simulation and measurement are in very good agreement in spite of the large uncertainty of the measurement of the dye's two-photon absorption close to the edge of the exciting spectrum. The contrast, defined in Eq. 4, is zero if the fluorescence signals of both dyes are equally large. In our case the Rhodamine B fluorescence was larger than the Coumarin 1 fluorescence due to different efficiency of the excitation. This leads to a contrast larger than zero. The contrast 
a) 3rd-order phase

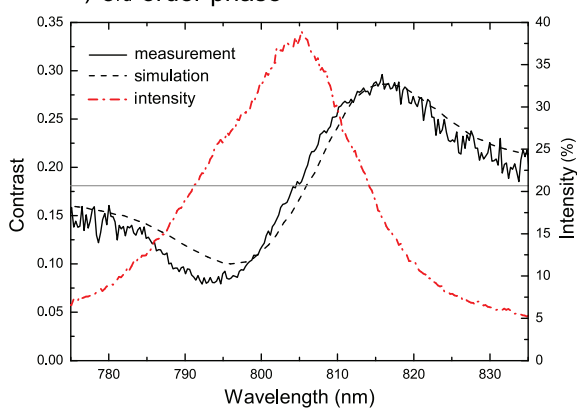

b) $\pi$-step

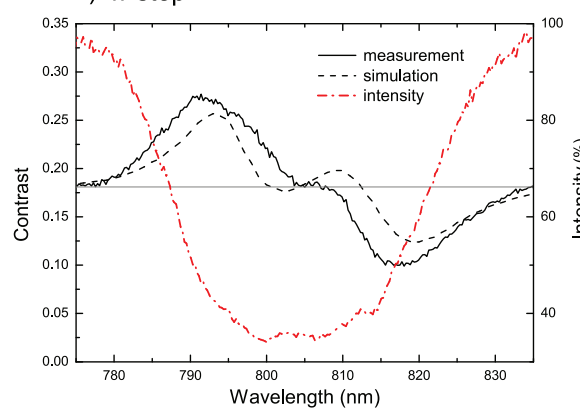

c) sinus-phase

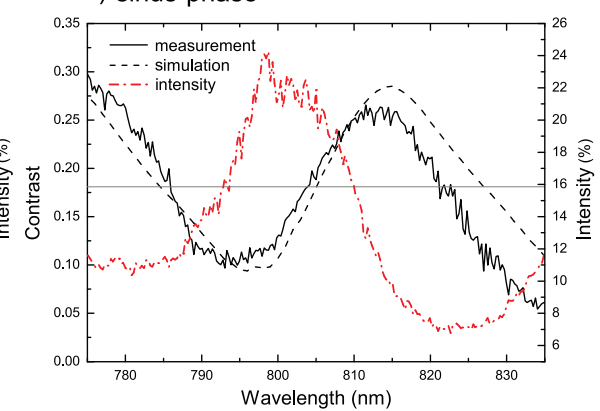

Fig. 4 Contrast measurements and simulations of the Rhodamine B and Coumarin 1 fluorescence signal for different shifted spectral phases. The red line (dash-dot) shows the corresponding measured fluorescence intensity. These measurements were done for precompensated pulses which were transmitted through the hollow-core fiber and then focused in a cuvette holding the dye solution. Third-order phase (a) of $2 \times 10^{5} \mathrm{fs}^{3}$ shifted from $775 \mathrm{~nm}$ to $835 \mathrm{~nm}$. The $\pi$-step phase (b) function has the lowest capabilities of optimizing the contrast in this comparison. The sine phase function (c) has an amplitude of 25 rad and $k=0.0736 \mathrm{~nm}^{-1}$. Achieved contrasts are comparable to those of the third-order phase function.

reached by the transform-limited pulse $\left(C_{T L}=0.18\right)$ is marked as a grey line and the fluorescence intensity relative to the TL pulse is shown in percent on the right axis. While all phase scans reach good contrasts between the two dyes, only the $\pi$-step function also shows large fluorescence (45\% for maximum and $52.5 \%$ for minimum contrast).

Fig. 5 shows the actual changes in fluorescence spectra. The spectrum was measured for pulses achieving both maximal and minimal contrast. The spectra were normalized because of the strong variations in intensities when changing the phase positions.

It can easily be seen that for each combination of dyes and every measurement setup one has to find an individual phase which generates optimal contrast while preserving a minimum of signal to noise ratio. Therefore we will now look at possible parametrizations best

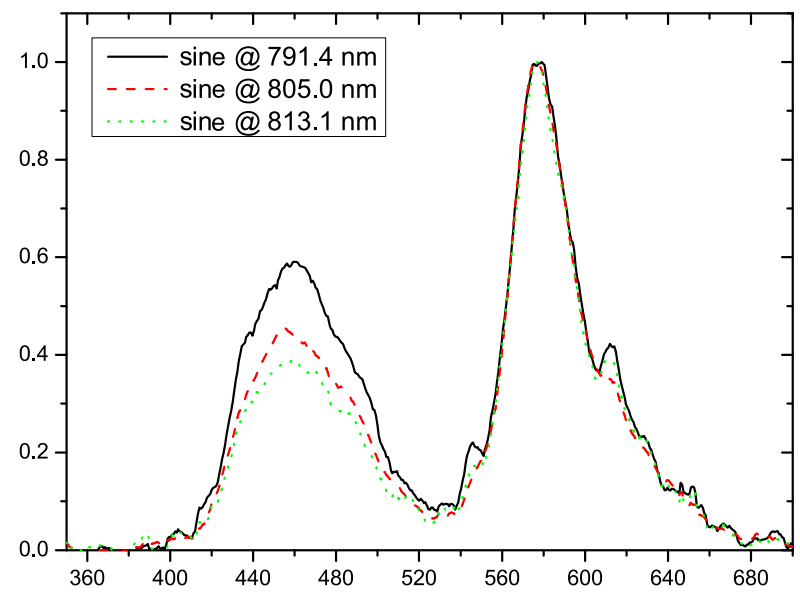

Fig. 5 Spectra measured for shifted phases from Fig. 4 from which the contrasts were computed. The sine phase was centered around wavelengths of maximal contrast deviation and around the central wavelength. The contrast measured was 0.11 at $791.4 \mathrm{~nm}$ and 0.26 at $813.1 \mathrm{~nm}$.

used in optimizations and outline the space of achievable contrasts. 


\section{Optimizations}

We tested different parametrizations regarding their effectiveness in optimizing the contrast using an evolutionary algorithm. As before we added a previously determined offset phase to compensate for the dispersion of the hollow-core fiber. While optimizing the contrast in favour of Coumarin 1 fluorescence we had to ensure that the algorithm did not reduce the fluorescence signal to zero. The optimization would favour this solution because the background signal gives a zero contrast. Therefore we normalized $I_{R}$ and $I_{C}$ seperately by the signal of the transform-limited pulse to make sure we get negative contrasts when optimizing Coumarin 1 and positive contrasts when optimizing Rhodamine B. This normalization was only applied when optimizing while all shown data shows unnormalized contrasts. We performed more than ten optimizations for each parametrization to rule out convergence to local minima. Due to the intrinsic problems of this two.goal optimizations it is hard to find one best parametrization. Because of that we optimized only the contrast and for now neglected the intensities.

The best solutions for each parametrization are shown in Table 2 and Table 3 for maximized and minimized contrast respectively. While the shifted third-order phase uses only two parameters and therefore converges very quickly, the high contrast deviations achieved come with a very low signal. The sine-phase adds one more parameter to the optimization and achieves nearly the same con- trast at a much higher fluorescence signal being thereby superior to the third-order phase parametrization.

The fifth-order polynomal phase parametrization converges most slowly but results in the best contrasts at intensities slightly above those of the shifted third-order phase. This phase function is hence favourable when searching for maximal contrasts. Fig. 6 shows the simulated two-photon field for the optimal phases presented in Table 2 and 3. The fifth-order polynomal parametrization allows for constructive interference on one side of the spectrum while interfering destructively on the other. Adding more phase terms to the parametrization did not increase the contrast significantly over the results obtained by the fith-order polynomal. Fig 6 shows as well that the sine-phase can be used when high fluorescence signals are required, e.g. when higher intensities would lead to sample damage or the fluorescence light cannot be easily collected. For such cases Ogilive et al. [7] showed that one can digitally enhance contrasts further by linear combination of captured images illuminated by both optimized pulses seperately.

\section{Solution set}

Due to the wide variety of phase functions found in other publications it is desireable to get an overview of possible parametrizations to identify their effectivity for a certain application. Two conflicting goals have been found: Either one can achieve high intensities but low contrast deviations or high selectivity while sacrifying the fluo- 
Table 2 Optimal phase parameters for maximizing the contrast (larger Rhodamine B fluorescence). As a reference: The transform-limited pulse leads to a contrast of 0.18 .

\begin{tabular}{lccl}
\hline parametrization & best contrast & intensity & parameters \\
\hline$A \sin \left(k\left(\lambda-\lambda_{c}\right)\right)$ & 0.28 & $22.37 \%$ & $\boldsymbol{A}=17.2 \mathrm{rad}, \boldsymbol{k}=0.059 \frac{1}{\mathrm{~nm}}, \boldsymbol{\lambda}_{\boldsymbol{c}}=813 \mathrm{~nm}$ \\
$\frac{b_{3}}{6}\left(\omega_{c}-\omega\right)^{3}$ & 0.32 & $4.1 \%$ & $\boldsymbol{b}_{\mathbf{3}}=7.94 \times 10^{5} \mathrm{fs}^{3}, \boldsymbol{\lambda}_{\boldsymbol{c}}=823 \mathrm{~nm}$ \\
$\sum_{i=2}^{5} \frac{b_{i}}{i !}\left(\omega_{c}-\omega\right)^{i}$ & 0.33 & $4.72 \%$ & $\boldsymbol{b}_{\mathbf{2}}=-3.84 \times 10^{4} \mathrm{fs}^{3}, \boldsymbol{b}_{\mathbf{3}}=-1.46 \times 10^{6} \mathrm{fs}^{3}$ \\
& & & $\boldsymbol{b}_{\mathbf{4}}=-5,23 \times 10^{5} \mathrm{fs}^{4}, \boldsymbol{b}_{\mathbf{5}}=1.35 \times 10^{9} \mathrm{fs}^{5}$ \\
\hline
\end{tabular}

Table 3 Optimal phase parameters for minimizing the contrast (larger Coumarin 1 fluorescence).

\begin{tabular}{lccl}
\hline parametrization & best contrast & intensity & parameters \\
\hline$A \sin \left(k\left(\lambda-\lambda_{c}\right)\right)$ & 0.09 & $22.99 \%$ & $\boldsymbol{A}=58.1 \mathrm{rad}, \boldsymbol{k}=0.037 \frac{1}{\mathrm{~nm}}, \boldsymbol{\lambda}_{\boldsymbol{c}}=791 \mathrm{~nm}$ \\
$\frac{b_{3}}{6}\left(\omega_{c}-\omega\right)^{3}$ & 0.08 & $8.8 \%$ & $\boldsymbol{b}_{\mathbf{3}}=8.89 \times 10^{5} \mathrm{fs}^{3}, \boldsymbol{\lambda}_{\boldsymbol{c}}=792 \mathrm{~nm}$ \\
$\sum_{i=2}^{5} \frac{b_{i}}{i !}\left(\omega_{c}-\omega\right)^{i}$ & -0.004 & $10 \%$ & $\boldsymbol{b}_{\mathbf{2}}=-3.79 \times 10^{4} \mathrm{fs}^{2}, \boldsymbol{b}_{\mathbf{3}}=1.67 \times 10^{6} \mathrm{fs}^{3}$ \\
& & & $\boldsymbol{b}_{\mathbf{4}}=-4.33 \times 10^{7} \mathrm{fs}^{4}, \boldsymbol{b}_{\mathbf{5}}=4.79 \times 10^{8} \mathrm{fs}^{5}$ \\
\hline
\end{tabular}

rescence intensity. In Fig. 7 the intensities corresponding to the previously shown phase scans are plotted over the contrast and compared to simulations. The transformlimited pulse has a contrast of 0.18 and serves as a reference for the intensities.

It is interesting to see that all results appear to lie in a triangle shaped solution space, i.e. the pareto-optimal front follows $\frac{d I_{f l}}{d C}=$ const. $>0$ for optimized Coumarin 1 signal and $\frac{d I_{f l}}{d C}=$ const. $<0$ for contrasts larger than the one of the transform-limited pulse. The slight differences between the simulations and the measured data are due to the uncertainty of two-photon absorbance of both dyes at the sides of the spectrum. Again the $\pi$-step phase provides large intensities at acceptable contrasts.
Because the $\pi$-step phase is described by only one parameter an optimization is not necessary. A simple scan can be used to find the optimal phase. If larger contrasts are required an optimization using multiple phase terms should be done.

Finally we tried to check if there are possible other phases which would outperform the ones tested in this paper. Therefore we created random phases and simulated their contrast and intensity. Using the domination principle we discarded any phase giving worse results in both goals (optimal contrast, high intensity) in comparison to all other randomly generated phases $[13,14]$. Keeping only phases not being dominated in both goals by all other phases in the regarded set of phases min- 

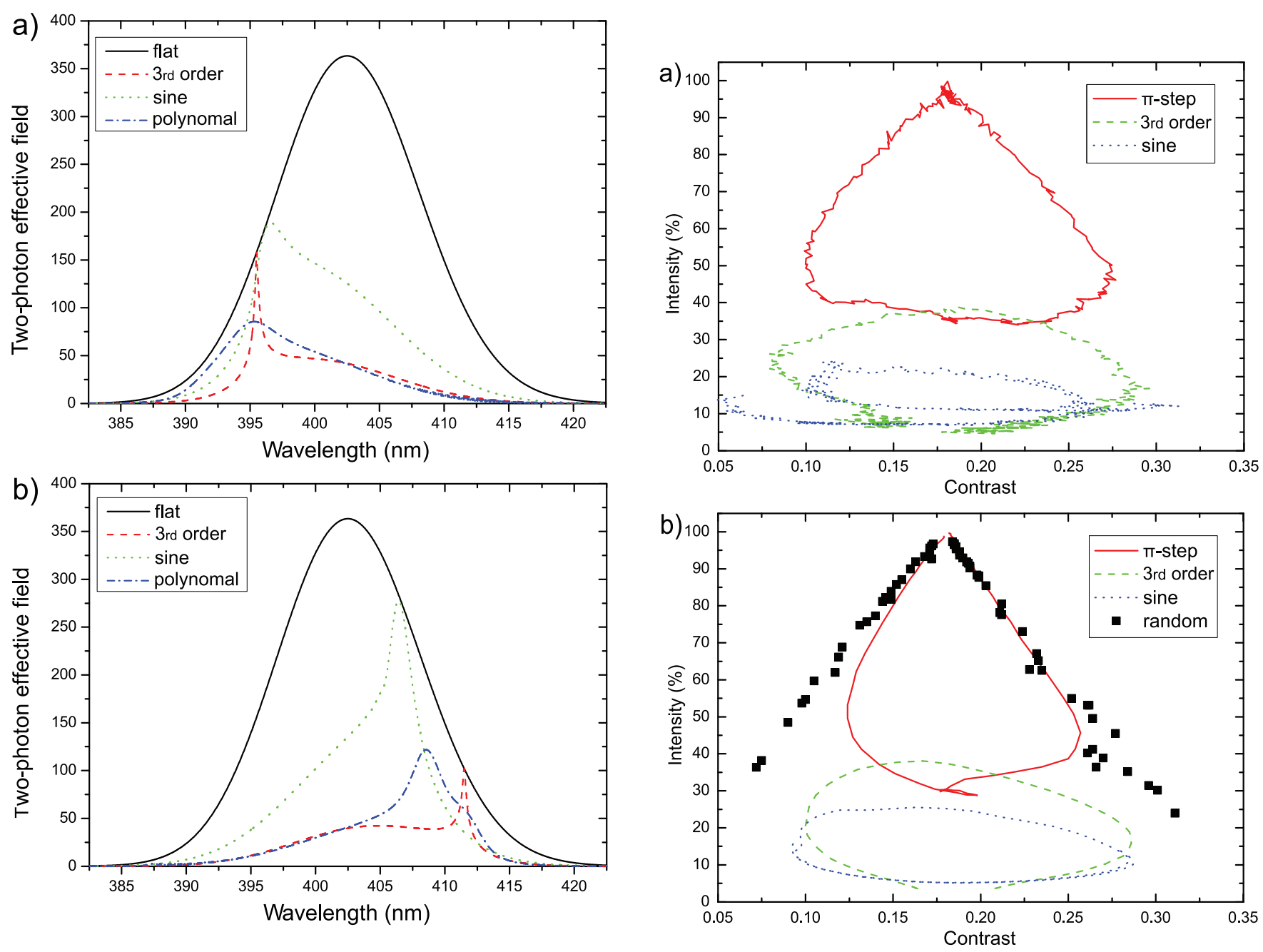

Fig. 6 a) Simulated two-photon field for the optimal phase functions shown in Table 3. The simulations show why the sine phase generates large fluorescence but low contrast. The third-order phase enables constructive interference in a very localized spectral range resulting in a very low fluorescence intensity. The most effective phase is the fifth-order polynom optimzation which leads to constructive interference for all wavelengths below $395 \mathrm{~nm}$. b) shows the simulated twophoton fields for optimal phase functions shown in Table 2 which maximizes the contrast in favour of Rhodamine B fluorescence.

Fig. 7 Intensity plotted over contrast for different spectral phase functions shifted along the pixels of the pulse shaper.

a) Experimental data b) Simulations derived as described earlier. Additionally Simulations of intensity and Contrast for randomly generated phase functions are shown which sketch out the available solution space when optimizing fluorescence intensity versus fluorescence contrast.

imizes overhead and after thousands of tested phases leaves the pareto-optimal results which are satisfying both goals. The calulated results are shown as black squares in Fig.7 and indicate that there is further improvement possible for low intensities while the $\pi$-step 
closely follows the pareto-optimal front at high intensities.

\section{Conclusion}

In this paper we demonstrated the possibility of transmitting precompensated phase-shaped pulses through a hollow-core photonic crystal fiber allowing for selective excitation of dye molecules in a solution. This could be used in endoscopic applications or in two-photon microscopy. Hollow-core fibers are used to transmit the large pulse energies of $35 \mathrm{~nJ}$ which are required for efficient two-photon excitation. These pulse energies could even be exceeded by eliminating the glass window in front of the fiber. Evolutionary optimizations as well as phase scans were used to identify optimal parametrizations for either maximal contrast or large fluorescence intensities. We showed that while multi-parameter optimizations give better contrast ratios a simple $\pi$-step phase can outmatch these phases when high fluorescence signals are required. (*This might be beneficial when the applied dyes possess a low two-photon fluorescence efficiency while in the case of Rhodamin B and Coumarin 1 one would choose phases optimizing the contrast since their fluorescence can still be easily collected.*) The phase scans were $\left(*\right.$ then $\left.^{*}\right)$ compared to simulations resorting to the previously measured two-photon absorption of Rhodamine $\mathrm{B}$ and Coumarin 1 in the relevant spectral region. Finally we sketched the solution set and showed what contrasts and intensities could be expected of other phase-functions not treated in this paper. The method demonstrated in this paper could in future be used for endoscopic applications (as in [19]) or be integrated in state of the art two-photon microscopes which would thereby benefit from the additional spectral sensivityand easy pulse delivery to the sample (as in $[15,16]$ ).

We thank Prof. Dr. Ludger Wöste for his support.

\section{References}

1. R.Heintzmann, G.Ficz, Briefings in Functional Genomics \& Proteomics 5, (2006) 289-301

2. F.Helmchen, W.Denk, Nature Methods 2, (2005) 932-940

3. D.Meshulach, Y.Silberberg, Physical Review A 60, (1999) $1287-1292$

4. W.Denk, J.H.Strickler, W.W.Webb., Science 248, (1990) 73-76

5. R.S.Pillai, C.Boudoux, G.Labroille, N.Olivier, I.Veilleux, E.Farge, M.Joffre, E.Beaurepaire, Optics Express 17, (2009) 12741-12752

6. J.M.Dela Cruz, V.V.Lozovoy, M.Dantus, Journal of Photochemistry and Photobiology A: Chemistry 180, (2006) 307-313

7. J.P. Ogilvie, D. Débarre, X.Solinas, J.-L.Martin, E.Beaurepaire, M.Joffre, Optics Express 14, (2006) 759-766

8. I.Pastirk, J.Dela Cruz, K.Walowicz, V.Lozovoy, M.Dantus, Optics Express 11, (2003) 1695-1701

9. M.Pawłowska, A.Patas, G.Achazi, A.Lindinger, Optics Letters 37, (2012) 2709-2711

10. G.Achazi, A.Patas, F.Weise, M.Pawłowska, A.Lindinger, Applied Optics 50, (2011) 915-923 
11. M.Pawłowska, A.Patas, G.Achazi, N.Rahmat, F.Weise, A.Lindinger, Journal of the Optical Society of America B 29, (2012) 833-840

12. J.K.Ranka, A.L.Gaeta, A.Baltuska, M.S.Pshenichnikov, D.A. Wiersma, Optics Letters 22, (1997) 1344-1346

13. K.Deb, Evolutionary Computation 7, (1999) 205-230

14. C.M. Fonseca, P.J. Fleming, Evolutionary Computation 3, (1995) 1-16

15. K.Svoboda, W.Denk, D.Kleinfeld, D.W.Tank, Nature 385, (1997) 161-165

16. C.Stosiek, O.Garaschuk, K.Holthoff, A.Konnerth, Proceedings of the National Academy of Sciences 100, (2003) 7319-7324

17. C.Xu, R.M.Williams, W.Zipfel, W.W.Webb, Bioimaging 4, (1996) 198-207

18. D.Bird, Min.Gu, Optics Letters 28, (2003) 1552-1554

19. Y.Zang, M.L.Akins, K.Murari, J.Xi, M.Li, K.LubyPhelps, M.Mahendroo, X.Li, Proceedings of the National Academy of Sciences 109, (2012) 12878-12883 